\title{
Assessment of Different Strategies in Optimizing Network Operation Incorporating PV System
}

\author{
Zen L. Chai ${ }^{1}$, S.P Ang ${ }^{1}$, A. Khalil ${ }^{1}$, M. A. Salam ${ }^{2}$, William Voon ${ }^{3}$ \\ ${ }^{1}$ Electrical and Electronic Engineering Programme Area, Universiti Teknologi Brunei, Brunei Darussalam \\ ${ }^{2}$ Department of Electrical and Computer Engineering, The University of Western Ontario, Canada \\ ${ }^{3}$ Berakas Power Management Company Sdn Bhd, Brunei Darussalam
}

\begin{tabular}{l} 
Article Info \\
\hline Article history: \\
Received May 20, 2019 \\
Revised Nov 29, 2019 \\
Accepted Dec 06, 2019 \\
\hline Keyword: \\
Distributed installation \\
reactive power control \\
network topology \\
loss reduction \\
voltage enhancement \\
solar photovoltaic
\end{tabular}

\begin{abstract}
Renewable distributed generation is increasingly deployed in distribution networks for meeting the rapidly-growing electricity demand and energy transition target. Its optimal integration could maximize the benefits in network operation and eliminate technical challenges to passive networks associated with its non-dispatchable generation characteristic. In this paper, various scenarios based on three different optimization strategies viz. i) distributed installation, ii) power factor and iii) network configuration are assessed. The optimization goals are minimizing active line losses and improving network voltage profile within the constraints. The analysis considers PV system integration, and the base configuration of centralized PV system installation is taken as the reference for comparison. Time-series load flow algorithm utilizing average PV system generation and load demand profiles is adopted in solving the multi-objective optimization problem with index weighting factors. A real $11 \mathrm{kV}$ distribution network in Brunei is modeled as the test system and integrated with the scenario-based PV system. The variations in generation and demand are considered in the work. The findings present the opportunities in furthering network operation enhancement with the distributed installation strategy having the highest potential. The analysis provides a clear optimization potential of each scenario, which shall be beneficial to the utility in planning new deployment.
\end{abstract}

Copyright $\odot 2019$ Institute of Advanced Engineering and Science. All rights reserved.

\section{Corresponding Author:}

Zen L. Chai,

Electrical and Electronic Engineering Department, Faculty of Engineering,

Universiti Teknologi Brunei,

Brunei Darussalam,

Email: zenl.chai@outlook.com

\section{INTRODUCTION}

The global electricity demand is growing at a rapid pace. Additional generation capacity is necessitated to be made available to cater the needs. Besides, the electricity sector is in the phase of energy transitioning towards a more sustainable way of operation. Within this context, the concept of distributed generation (DG) involving renewable energy (RE) technology such as solar photovoltaic (PV), wind and biomass is increasingly introduced as the alternative generation source in the electrical grid. Recognizing the RE target set in worldwide, a significant share of renewable DG in the electricity generation mix is foreseeable. Among the myriad of RE technologies available, the PV system has gained wide interests and a rising uptake due to its continuous price reduction and technological advancement [1]. Additionally, the utilization of free and abundant solar insolation as its input and the features of carbon-free, minimal maintenance and noiseless operation make the PV system a preferred choice of DG.

However, the integration of DG based on the PV system in the traditional electrical grid is a challenging and complex task. This is owing to the intermittent and non-dispatchable generation characteristics of the PV system. Furthermore, the concern lies in the fact that most distribution networks in operation today are designed as a passive system. Only unidirectional power flow from the main generation station to the loads 
is expected. Consequently, the usual power flow direction may be altered with the increase of PV system capacity in the network. The integration of the PV system in the network may bring about positive or negative impacts. Previous studies [2-6] have shown that various network operational limits may be violated due to the generation and demand mismatch arising from the integration. Examples of the violations are bus overvoltage, line and transformer overloading, and possibly leading to an increase in network losses. Despite that, the strategic integration of the PV system shall contribute to the network operation. The technical benefits may include network loss reduction, voltage profile improvement, increase in network loadability, and improve in sustainability in terms of minimized carbon emission and usage of fossil fuels [2, 6]. In this regard, a comprehensive analysis and proper planning are imperative for ensuring that the network stability and reliability are maintained while leveraging the advantages from the integration.

The optimization of network operation by utilizing renewable DG has attracted wide interests among the researchers. There have been numerous attempts in employing different optimization strategies for achieving pre-specified goals using various approaches. Optimal allocation of DG was proposed in numerous studies. The strategy was employed by Ghosh et al. [7] for a 33-bus network for voltage profile enhancement and system loss minimization through a load flow algorithm. Jamil and Anees [8] identified the optimal size and location of multi-location DG in IEEE 33 and 69 bus networks for improving voltage profile, reducing losses and optimizing the costs using an analytical method. The optimal allocation of three types of DG for loss reduction was proposed by Prenc et al. [9] while accounting average daily electricity generation and load profile. Liu et al. [10] searched for the optimal DG location and capacity based on time-varying generation and demand for achieving the multi-objective goals involving economic and technical factors.

The analysis by Hung et al. [11] involved assessing the optimal power factor of DG for loss minimization using analytical approaches, in addition to the optimal location and size. The strategy of leveraging the power factor control capability of solar inverter was proposed by Abdelmotteleb et al. [12]. The work aimed for reducing losses and maximizing line and feeder reserve capacity in an IEEE 34 bus system. Alternatively, Bouhouras et al. [13] studied the network configuration strategy for loss reduction in 16, 33 and 39 bus grid systems integrated with the PV system by utilizing spatial and quantitative sensitivity study. Murthy et al. [14] examined the optimal network configuration with the aim of minimizing the losses and enhancing the voltage profile in a 33-bus system with DG.

Though various types of strategies are available, it is hypothesized that they possess dissimilar optimization potential. Yet, the comparison between their potentials has not much been assessed in a unified network model and in the perspective of optimizing in comparison to a proposed PV system configuration with a specified cumulative installed capacity. It is also worth noting that numerous good earlier works solved the optimization problem in consideration of the operating condition at a specific point in time, such as maximum DG generation and maximum loading only [7, 8, 14-17]. Thereby, the influence on the results due to the condition at other times may be understated. The variations in generation and demand in the network are required to be considered for improved accuracy $[11,18]$.

In this paper, various scenarios based on three different optimization strategies viz. i) distributed installation, ii) power factor and iii) network configuration are focused on assessing their potentials in optimizing the network operation, in comparison with the PV system of base configuration. Time-series load flow algorithm utilizing average PV system generation and load demand profiles is adopted as the method, and the generation and demand variations are considered. The problem is formulated as a multi-objective optimization problem with the goals of minimizing active line losses and enhancing voltage profile in the network. A real $11 \mathrm{kV}$ distribution network in Brunei is modeled and used as the test system with the integration of the PV system.

\section{METHODOLOGY AND MODELING}

Given the background, the work will commence with deriving the time-series potential generations of the PV system modeled for each scenario. The next step will encompass the modeling of the $11 \mathrm{kV}$ distribution network of Brunei and the load demands. The scenario-based integration of the PV system will then be carried out. The analysis will proceed with assessing the network operation enhancement potential of each scenario within a set of constraints using a multi-objective performance index.

\subsection{PV system and distribution network}

PVsyst software is utilized for modeling the PV system. PVsyst is a well-recognized software package specializing in PV system design and sizing. In the present work, the cumulative PV system installed capacity is specified as $2 \mathrm{MW}$. The system is formed by 17,390 units of $115 \mathrm{~W}$ thin-film cadmium telluride (CdTe) PV panels manufactured by First Solar. The panels are fixed to orient due South and tilted at $10^{\circ}$. A solar inverter is an essential component in a PV system for converting direct current power generated into alternating current (AC) power useable in the network. The specification of an inverter varies across different manufacturers and 
models. Here, the inverter selection is limited to the product manufactured by SMA and within the product family range of Sunny Central CP XT. These limitations shall ensure a minor performance and capability variations between the inverters of different capacities. The inverter is chosen based on the closest available nominal AC power rating in matching the PV system installed capacity in each scenario. Table 1 shows the specified nominal AC power rating of the inverter with respect to each PV system installed capacity.

Table 1. Specified rating of solar inverter.

\begin{tabular}{cc}
\hline Inverter rated power (MVA) & $\begin{array}{c}\text { System installed capacity } \\
(\mathrm{MW})\end{array}$ \\
\hline 0.50 & 0.58 \\
0.63 & 0.65 \\
0.72 & 0.77 \\
0.85 & 0.86 \\
0.90 & $0.92 / 0.94$ \\
1.00 & $1.00 / 1.06 / 1.08 / 1.14$ \\
$2 * 1.00$ & 2.00 \\
\hline
\end{tabular}

The main factors dictating the energy generation from a PV system are solar irradiance and ambient temperature at the installation site. Other factors include the environment, orientation, system component, and shading. In this work, Meteonorm software is employed to acquire the meteorological data needed for estimating the potential generations from the PV system. Meteonorm is a software package comprising of a comprehensive climate measurement database. It should be mentioned that a similar meteorological condition is assumed for all scenarios of the optimization strategies.

Taking the factors into account, the generations from the PV system over a year are computed in hourly time resolution in PVsyst for each scenario. Then, the sum of the generations for a specific hour is divided by the number of available data to determine the magnitude of the average PV system generation at the hour. The value of the peak generation in the year is also recorded. Figure 1 illustrates the hourly average generation of the PV system of base configuration normalized to the maximum value, which is utilized in the subsequent analysis. Using the similar method, the average curve and peak value of the generation for the PV system in other scenarios are derived.

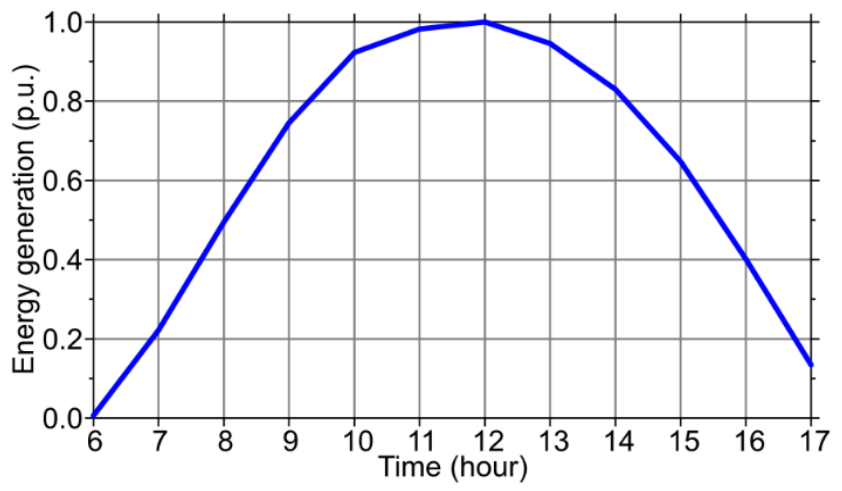

Figure 1. Average generation profile of PV system with base configuration.

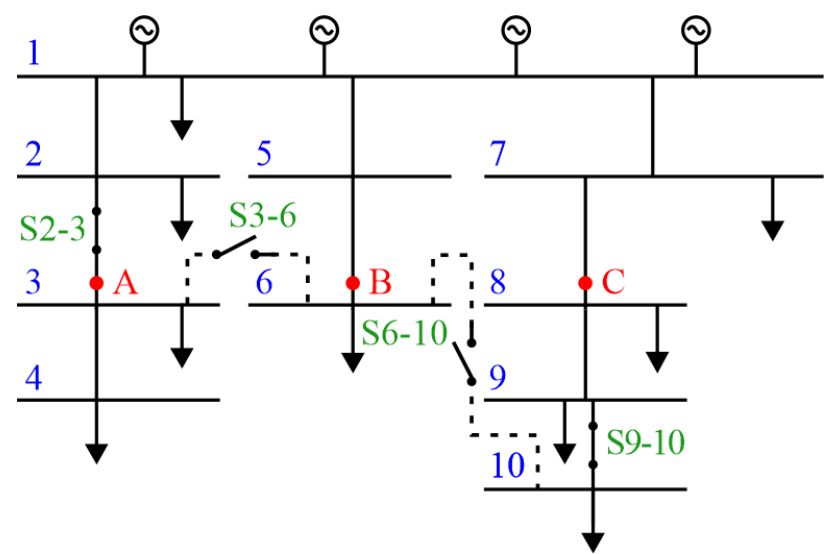

Figure 2. Single line diagram of the distribution network. 


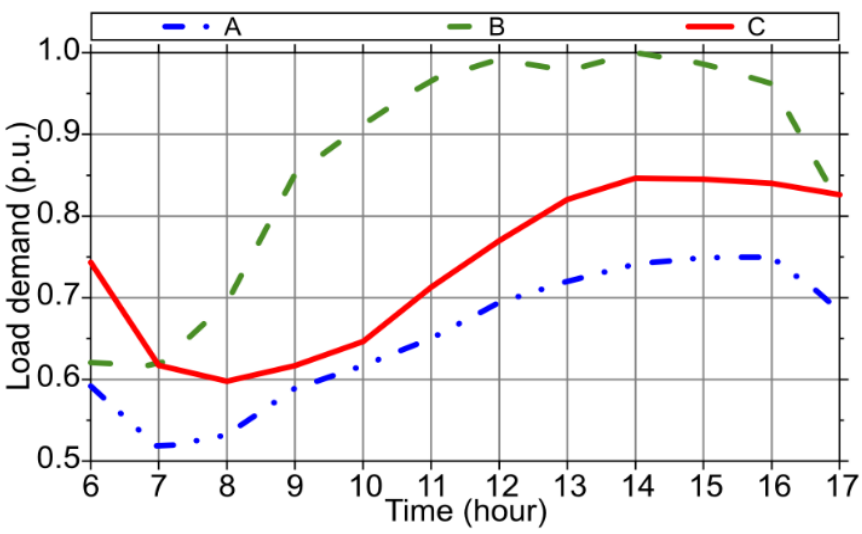

Figure 3. Average load demand profiles.

The single line diagram of the distribution network modeled in the present work is depicted in Figure 2. The network is a radial system and operates in passive mode. There are two types of switches which determine the network topology. A normally-closed switch is denoted as a sectionalizing switch, while a normally-open switch is known as a tie switch. Two sets of tie switches are available in the network for realizing the interconnection of bus 3 with bus 6 and bus 6 with bus 10 by energizing the lines. The status of the switch could be altered by the distribution network operator (DNO) for satisfying network operational requirements.

A proposed centralized PV system with an installed capacity of $2 \mathrm{MW}$ is envisaged for integrating at bus 6 , and it is denoted as the base configuration in the present work. The representative average profiles of the load demand measurements at points A, B and C normalized to the maximum value are shown in Figure 3. The average demand of each hour is derived from the division of the sum of the hour-specific demands obtained from long-term measurement with the total number of measurements. The peak and minimum demands during the measurement period are also acquired. These demand data are included in the modeling of the network.

In this work, there are several constraints concerning the PV system and the network, as detailed below:

(i) The operating bus voltages in the network must lie within the acceptable range as described in equation

(1). Here, the $V_{\min }$ and $V_{\max }$ are specified as 0.95 p.u. and 1.05 p.u, respectively.

$$
V_{\min } \leq V_{i} \leq V_{\max }
$$

where $V_{\min }=$ minimum allowable voltage at bus $i$ and $V_{\max }=$ maximum allowable voltage at bus $i$.

(ii) The network topology may be modified within the possibilities offered by the available switches. However, its radiality characteristic must be preserved and all the loads must remain energized.

(iii) Recognizing the geographical condition, the PV system integration is practically possible at bus 3, 6 and 8 only. In addition, the possibilities of integrating the proposed installed capacity of $2 \mathrm{MW}$ as a centralized installation (base configuration) at bus 3 and 8, respectively, are excluded due to certain factors. However, the PV system may be split into multiple smaller systems for integration as distributed installations in suiting the requirement in each scenario. In all the scenarios, the total PV system installed capacity in the network must remain as $2 \mathrm{MW}$ as defined in equation (2).

$$
\sum_{i=1}^{n} P_{P V, i}=2 \mathrm{MW}
$$

where $P_{P V, i}=\mathrm{PV}$ system installed capacity in MW at bus $i$ and $n=$ number of buses.

(iv) All the solar inverters must satisfy the operating power factor range of SMA Sunny Central CP XT inverter as given in equation (3). The $P F_{\text {min }}$ and $P F_{\text {max }}$ are limited to 0.90 leading and 0.90 lagging, respectively. In this analysis, the power factors of all the solar inverters are specified as 0.95 operating in a lagging mode, unless otherwise specified.

$$
P F_{\text {min }} \leq P F_{P V} \leq P F_{\text {max }}
$$

where $P F_{\text {min }}=$ minimum power factor limit of the inverter and $P F_{\max }=$ maximum power factor limit of the inverter. 


\subsection{Computational method}

Time-series load flow algorithm employing the Newton-Raphson (NR) method is adopted for solving the power flow problem of the network. In terms of convergence speed and accuracy, the NR method was proven to outperform other techniques [19]. The solution is determined by an iterative calculation process until a pre-specified accuracy is achieved. Equation (4) and (5) are the main mathematical expressions used in deriving the power flow solution.

$$
\begin{aligned}
& P_{i}=\sum_{k=1}^{n}\left|V_{i}\right|\left|V_{k}\right|\left(G_{i k} \cos \theta_{i k}+B_{i k} \sin \theta_{i k}\right) \\
& Q_{i}=\sum_{k=1}^{n}\left|V_{i}\right|\left|V_{k}\right|\left(G_{i k} \sin \theta_{i k}-B_{i k} \cos \theta_{i k}\right)
\end{aligned}
$$

where $n=$ number of buses, $V_{i}=$ voltage of bus $i, V_{k}=$ voltage of bus $k, G_{i k}=$ conductance between bus $i$ and bus $k, \theta_{i k}=$ phase angle and $B_{i k}=$ susceptance between bus $i$ and bus $k$.

In the computation process, bus 1 is assigned as the slack bus with a voltage magnitude of 1 p.u. The voltage magnitude drops across the electrical components such as line and transformer. The operating voltages of all the other buses are determined from the successive iterations. They are expressed in relative to the specified voltage of the slack bus. The detailed computation procedure using the NR method could be referred in [19]. The power transfer across the line involves losses due to line impedances. In the present work, the active component of the power loss in the line is focused. The active line losses are defined in equation (6).

$$
P_{\text {loss }}=\sum_{i=1}^{n} 3 \times I_{i}^{2} R_{i}
$$

where $i=$ line number, $n=$ number of lines, $I=$ current and $R=$ resistance of line $i$.

The method commences with performing preliminary load flows of the existing network without the PV system in corresponding to different loading levels (maximum and minimum). This step is essential for assessing the compliance of the existing network against the constraints. Any non-compliance throughout the solving process will lead to cessation of the algorithm until the issue is rectified. Next, the existing network with average demand profile is simulated and the resulting values are stored.

The peak generation of the PV system with base configuration and the minimum demand data are combined to represent the extreme condition. Load flow is conducted for the network integrated with the PV system of base configuration under the extreme condition. The resulting network operation is evaluated for its adherence with the constraints. Given no violation, the algorithm proceeds to forming a set of conditions comprising the average PV system generation profile of the base configuration and the average demand profile. Time-series load flow is then performed considering all the 12 hourly conditions for computing the active line losses and bus voltages in the network. The solutions are stored and expressed as a multi-objective performance index. Similarly, the procedure is repeated from assessing the compliance of the network with the PV system for all the scenarios of optimal distributed installation and optimal power factor strategy. As for optimal network configuration strategy, the procedure is repeated from the step prior to the PV system integration for assessing the compliance of the new network topology with the constraints before proceeding to the next step.

The aim of this analysis is to assess the potential of each strategy in optimizing the network operation, in comparison with the PV system of base configuration. The optimization goals are active line losses minimization and network voltage profile improvement. More efficient power transfer in the network is achieved with the reduction in line losses. Moreover, a better voltage profile suggests a lower risk of voltage limit violation and an improvement of system loadability. Two performance indices viz. active line losses index $(A L L I)$ and voltage index $(V I)$ are utilized for the assessment. The $A L L I$ is formulated in equation (7) where a more effective loss minimization is reflected by a lower magnitude of $A L L I$.

$$
A L L I=\sum_{t=1}^{h} P_{l o s s, t}^{\text {new }} / \sum_{t=1}^{h} P_{\text {loss }, t}^{\text {old }}
$$

where $P_{\text {loss }, t}^{\text {new }}=$ total active line losses in the network with PV system during hour $t, P_{\text {loss }, t}^{\text {old }}=$ total active line losses in the existing network prior to having PV system during hour $t$ and $h=$ number of hours.

The $V I$ is determined by the mathematical expression defined in equation (8). A smaller VI indicates a lesser average deviation from the nominal bus voltage.

$$
V I=\sum_{t=1}^{h}\left|\frac{V_{n o m}-V_{b, t}}{V_{n o m}}\right|_{b=2}^{n b} /(h \times n b)
$$

where $V_{n o m}=$ nominal bus voltage magnitude, $V_{b, t}=$ voltage magnitude of bus $b$ at hour $t, n b=$ number of buses and $h=$ number of hours. The voltage magnitude at the slack bus is not considered in the computation as it is pre-specified as a constant value. 
The problem is formulated as a multi-objective optimization problem. In solving the problem, each performance index is assigned with a weighting factor $\omega$. The multi-objective performance index is determined by the summation of all the performance indices along with their individual $\omega$ as given in equation (9). The value of $\omega$ can be varied by DNO according to the significance of each index in contributing to the network operation enhancement. Nevertheless, the conditions in equation (10) and (11) must be fulfilled. Here, $\omega_{1}$ and $\omega_{2}$ are set as 0.60 and 0.40 , respectively. The allocation put a higher emphasis on loss minimization compared to voltage profile improvement in solving the optimization problem.

$$
\begin{aligned}
& z=\omega_{1} A L L I+\omega_{2} V I \\
& 0 \leq \omega_{i} \leq 1 \\
& \sum_{i=1}^{2} \omega_{i}=1
\end{aligned}
$$

term $i$.

where $\omega_{1}=$ weighting factor for $A L L I, \omega_{2}=$ weighting factor for $V I$ and $\omega_{i}=$ weighting factor of the

The objective function is established as minimizing the multi-objective performance index as described in equation (12), subjected to the specified constraints.

$$
f=\operatorname{minimize}(z)
$$

The $z$ will be derived for all the scenarios. The minimum $z$ indicates the highest potential in minimizing the active line losses and enhancing the network voltage profile.

\section{RESULTS AND DISCUSSION}

In the present work, the network operating condition is computed by using load flow algorithm. The existing network without the PV system is assessed for compliance with the operational limit. The maximum and minimum network loading conditions are considered as the network may be risked to undervoltage and overvoltage issue, respectively. Table 2 shows the operating bus voltages in the network are within the range of 0.95 p.u. and 1.05 p.u. during the maximum and minimum demand. The results proved that no limit violation is presented in the existing network under the normal operating condition.

Table 2. Bus voltages during maximum and minimum demand in the existing network.

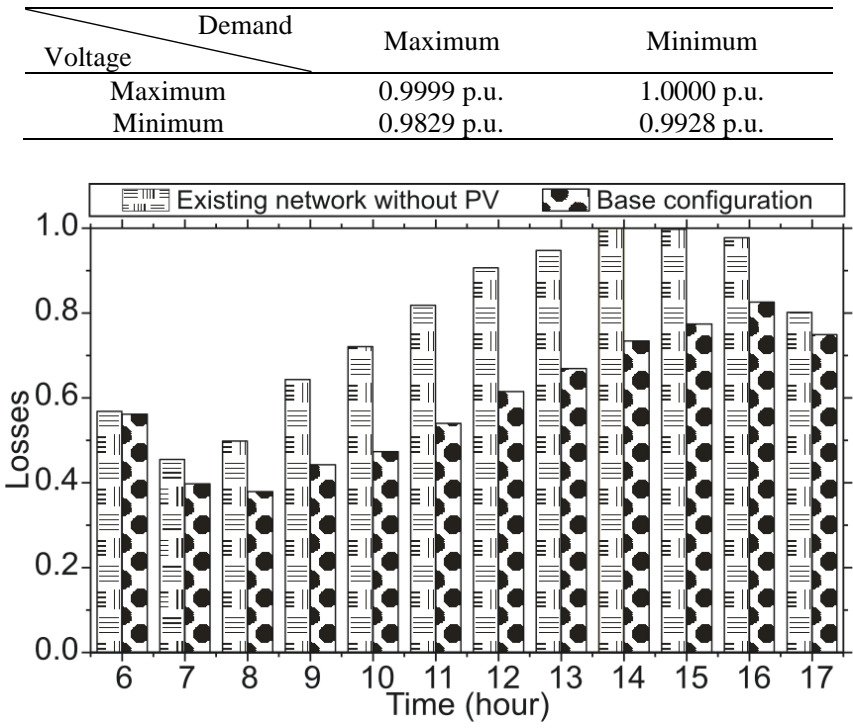

Figure 4. Hourly total active line losses before and after the PV system integration of the base configuration.

\subsection{Base configuration}

In this scenario, the PV system is integrated as a centralized installation at bus 6 with the installed capacity of $2 \mathrm{MW}$. The network conforms to the operational limit during peak PV system generation at the minimum demand. Figure 4 illustrates the total active line losses for each hour in the existing network versus those of after the PV system integration in the base configuration normalized to the maximum value. They are computed based on the average PV system generation and load demand profile. It is obvious that the losses are 
reduced for all the hours with the PV system in the network. For the time interval considered, the total active line losses in the network before and after the integration of the PV system are $348.5 \mathrm{~kW}$ and $267.3 \mathrm{~kW}$, respectively. In addition, the average network voltage is increased from 0.9955 p.u. to 0.9959 p.u. after having the PV system. It is proven from the results that the integration of PV system as DG is beneficial to the network as it is being a nearer source to the loads. A larger benefit to the network operation may be possible with an appropriate optimization strategy.

\subsection{Optimal distributed installation}

Eight scenarios comprising of different combinations of PV system capacity distribution and integration point are analyzed as summarized in Table 3. Scenario 1 to 4 represent the cases of equal distribution of the specified 2 MW PV system capacity. On the other hand, Scenario 5 to 8 consider a practical condition where the 2 MW PV system capacity distribution is assigned based on the total average load demand in each selected integration bus. In all the scenarios, the network operation does not violate the limit during peak PV system production at the minimum demand. Table 3 shows the comparison of the total active line losses and average network voltage between the scenarios, along with the description of the PV system integration. The obtained results are corresponding to the average PV system generation and load demand profile.

Table 3. Total active line losses and average network voltage of the distributed installation scenarios.

\begin{tabular}{|c|c|c|c|c|c|}
\hline \multirow{2}{*}{ Scenario } & \multicolumn{3}{|c|}{ PV system capacity (MW) } & \multirow{2}{*}{$\begin{array}{l}\text { Total active line losses } \\
(\mathrm{kW})\end{array}$} & \multirow{2}{*}{$\begin{array}{l}\text { Average network voltage } \\
\text { (p.u.) }\end{array}$} \\
\hline & Bus 3 & Bus 6 & Bus 8 & & \\
\hline 1 & 1 & 1 & 0 & 252.2 & 0.9962 \\
\hline 2 & 1 & 0 & 1 & 291.9 & 0.9962 \\
\hline 3 & 0 & 1 & 1 & 291.6 & 0.9958 \\
\hline 4 & 0.667 & 0.667 & 0.667 & 273.2 & 0.9961 \\
\hline 5 & 0.86 & 1.14 & 0 & 252.5 & 0.9962 \\
\hline 6 & 0.94 & 0 & 1.06 & 293.7 & 0.9962 \\
\hline 7 & 0 & 1.08 & 0.92 & 289.2 & 0.9958 \\
\hline 8 & 0.58 & 0.77 & 0.65 & 273.2 & 0.9961 \\
\hline
\end{tabular}

Based on Table 3, Scenario 1 is proven to be the optimal distributed installation in terms of active line losses reduction and network voltage profile improvement. In comparison with the PV system integration of the base configuration, the total active line losses are decreased from $267.3 \mathrm{~kW}$ to $252.2 \mathrm{~kW}$ in Scenario 1 , and the average network voltage is improved from 0.9959 p.u. to 0.9962 p.u. As a result, the efficiency and loadability of the network are further improved. This finding is in line with the previous study by Jamil and Anees [8] that the PV system deployment as distributed installation possesses the potential in providing more benefits to the network operation in terms of losses and voltage than centralized installation. It is worth pointing out that the results obtained in this work have taken the variations of generation and demand into account.

Instead of deploying the PV system as a centralized installation, distributed installation as in Scenario 1 provides more benefits to the network operation. The second optimal distributed installation is followed by Scenario 5. The results indicate that the potential of the PV system in further enhancing the network operation varies across the scenarios. In comparison with the integration of the base configuration, the total active line losses resulting from Scenarios 2, 3, 4, 6, 7 and 8 are risen. Despite that, their average network voltages are improved with exceptions to Scenarios 3 and 7. Thereby, the weighting factor of each performance index shall play an important role in evaluating the optimization potentials of these scenarios.

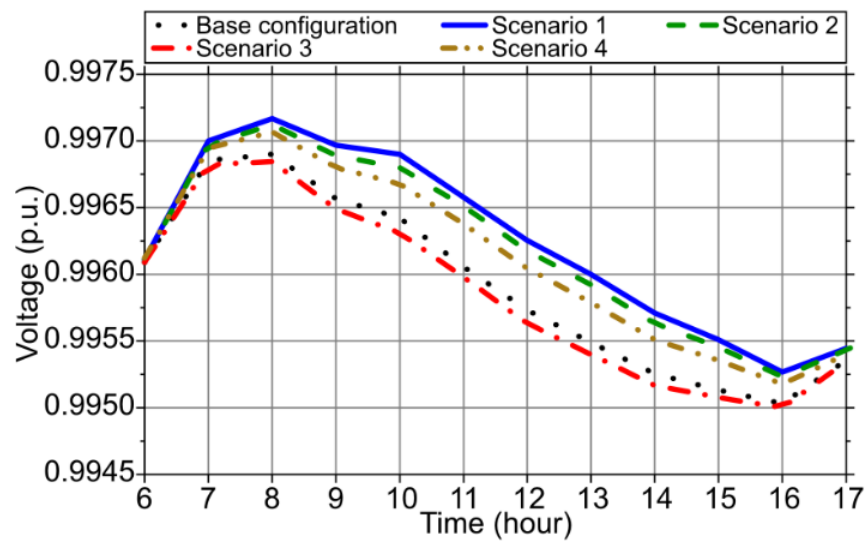

Figure 5. Average network voltage profile of each scenario. 
Figure 5 depicts the hourly average network voltage curves resulting from the PV system integration of the base configuration and Scenarios 1 to 4 . Similarly, the curves can be plotted for Scenarios 5 to 8 . Based on Figure 5, it can be noted that the average network voltage of Scenario 1 is higher than that of the base configuration for all the hours. It is worth noting that Scenario 3 presents a lower average network voltage than the base configuration for most of the hours. This demonstrates the contrasting impact between the PV system integration at the optimal and inappropriate points. Therefore, the optimal integration scenario is crucial to be identified in the planning stage for optimizing the benefits of the PV system in contributing to the network operation

\subsection{Optimal power factor}

The potential of leveraging reactive power control capability of the inverters in further enhancement of the network operation is investigated. The power factors of the inverters are varied within the specified operational constraint, ranging from 0.90 leading to 0.90 lagging in the step of 0.05 . The parameters and integration point of the PV system of base configuration are maintained for all the scenarios except the power factor of the inverter of which is varied according to the specified for each scenario as displayed in Table 4. In all the scenarios, the network complies with the operational limits under the extreme PV system generation and loading conditions.

Table 4. Power factor setting of the inverter

\begin{tabular}{ccc}
\hline Scenario & Power factor & Operating mode \\
\hline 1 & 0.90 & Lagging \\
2 & 0.95 & Lagging \\
3 & 1.00 & Unity \\
4 & 0.95 & Leading \\
5 & 0.90 & Leading \\
\hline
\end{tabular}

In Figure 6, the total active line losses and the average voltage of the network corresponding to the average profiles of the PV system production and load demand for each power factor setting of the inverter are illustrated. The results show that the losses are reduced as the inverter is set to operate increasingly towards the lagging mode. This implies that the reactive power injection into the network, in addition to the active power injection brings about a positive impact on the network operation. The finding is in good agreement with the work by Hung et al. reported in [11]. In addition to the losses, the present work includes considering the effect on the network voltages in evaluating the optimization potential.

Based on Figure 6, the optimal power factor of the inverter is determined to be 0.90 lagging for achieving the least total active line losses and the best average network voltage. In comparison with the base configuration, the total active line losses are reduced from $267.3 \mathrm{~kW}$ to $261.4 \mathrm{~kW}$, and the average network voltage is improved from 0.9959 p.u. to 0.99595 p.u. in Scenario 1. The results suggest the opportunity for the DNO in utilizing the in-built reactive power control capability of the inverter for further optimizing the network operation.

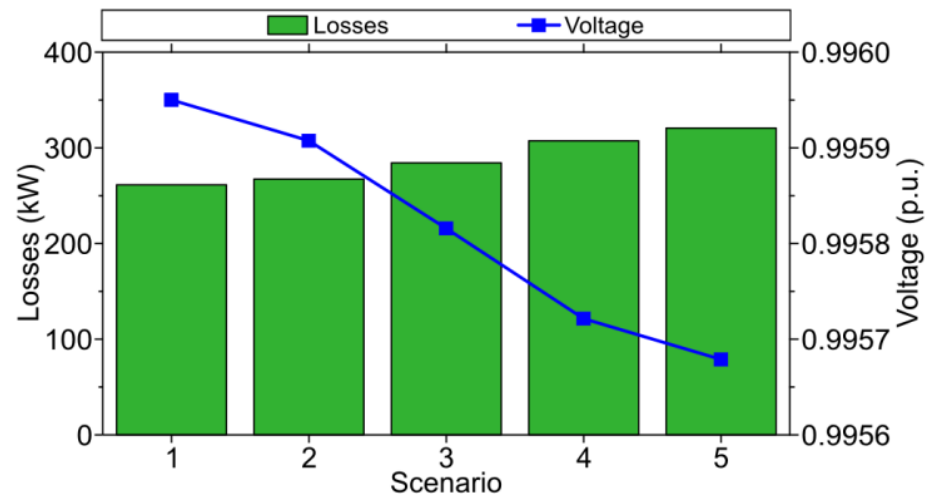

Figure 6. Total active line losses and average network voltage of the power factor setting scenarios

\subsection{Optimal network configuration}

In this strategy, all the possible network topologies are analyzed by altering the statuses of the switches available in the network. The PV system remains as the base configuration and integrated to bus 6 in all the scenarios. Table 5 summarizes the combination of switch status in each scenario. For every set of tie switch being closed, the corresponding set of the sectionalizing switch is opened for complying with the given constraint of preserving the network radiality. During the extreme condition, no operational limit violation is 
presented in the network with the resulting topology in each scenario in both before and after the integration of the PV system.

Table 5. Statuses of the network switches

\begin{tabular}{ccc}
\hline Scenario & Switch closed & Switch opened \\
\hline 1 & S3-6 & S2-3 \\
2 & S6-10 & S9-10 \\
3 & S3-6, S6-10 & S2-3, S9-10 \\
4 & S2-3, S9-10 & S3-6, S6-10 \\
\hline
\end{tabular}

Figure 7 displays the total active line losses and average network voltage of the four scenarios, considering the average PV system generation and demand profiles of the $11 \mathrm{kV}$ distribution network in Brunei. It is noted that in the case of deploying the PV system of base configuration, Scenario 4 represented by the existing network topology achieves the lowest total active line losses and the best average network voltage. This is followed by Scenarios 2, 1 and 3. Altering the network topology leads to the change in the power flow in the network. Consequently, the losses and voltage profile are impacted, as demonstrated in the results and the previous study based on a typical network model in [14]. Therefore, the optimization of the network topology in accommodating the PV system integration shall be performed during the planning phase for maximizing the benefits to the network operation.

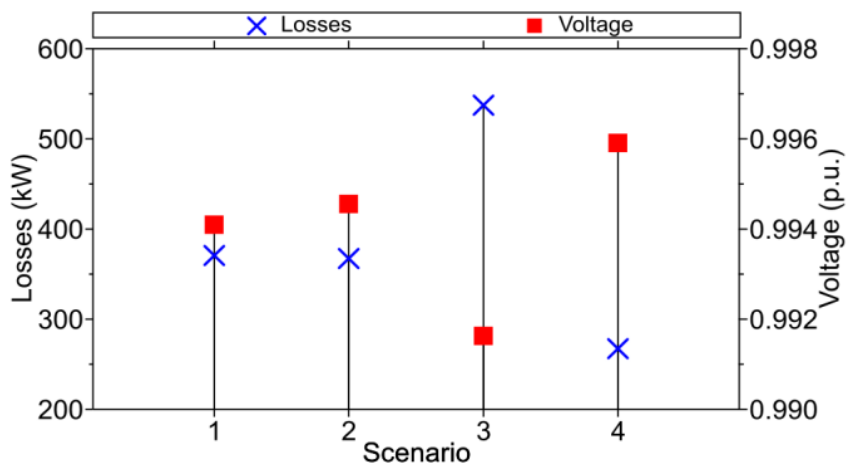

Figure 7. Total active line losses and average network voltage of the network configuration scenarios

\subsection{Comparisons of multi-objective performance index $(z)$}

The present work extends with a comparison of the optimization potentials of the scenarios corresponding to each strategy based on $z$ as derived using equations (7)-(9). Figure 8 presents the magnitude of $z$ determined for each scenario of the optimization strategies, in comparison with the $z$ of the base configuration. As mentioned, the scenario with the highest optimization potential is reflected by a minimum $z$. Among all the scenarios, Scenario 1 of the distributed installation strategy demonstrates the highest potential $(z=0.435742)$, followed by Scenario 5 of the distributed installation strategy $(z=0.436326)$ and Scenario 1 of power factor strategy $(z=0.451764)$. In fact, their $z$ values are lesser than the base configuration $(z=0.461917)$. The finding implies that the deployment of the PV system based on any of these three scenarios is more effective in terms of active line losses reduction and network voltage profile improvement compared to the base configuration.

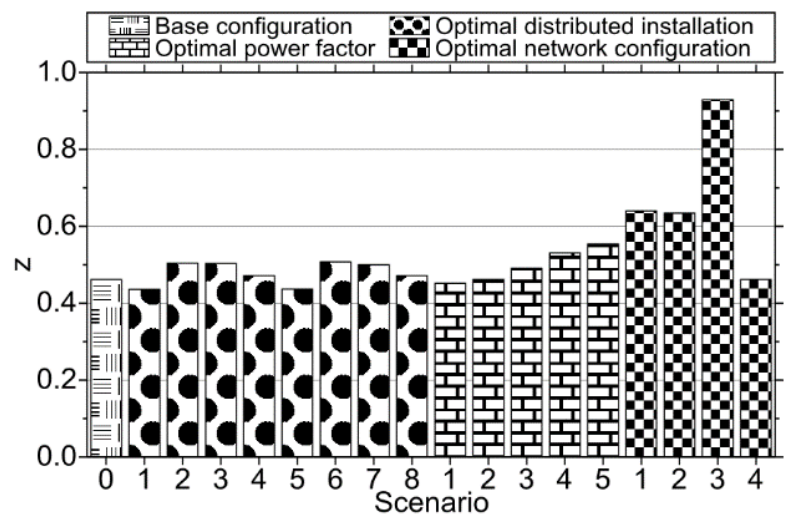

Figure 8. Multi-objective performance indices. 


\section{CONCLUSION}

In this paper, time-series load flow algorithm considering the average PV system generation and load demand profiles has been utilized for examining the potentials of the three different optimization strategies distributed installation, power factor and network configuration, in optimizing the network operation through reducing the active line losses and enhancing the voltage profile of the distribution network in Brunei. The benefits associated with integrating the PV system as DG in the network and the opportunities of furthering the network operation enhancement with the optimization strategies in comparison with the base configuration of centralized PV system deployment have been demonstrated in this paper. The highest optimization potential has been shown in the distributed installation strategy, followed by the power factor strategy and the network configuration strategy. Among the possible switching combinations analyzed, the existing topology of the network has been identified to be optimal for the PV system integration of the base configuration. This paper highlights the need for conducting the optimization assessment and considering different strategies for providing a clear outlook on the optimization potential of each strategy, which shall be beneficial to the DNO in planning the new PV system deployment. Future works shall be carried out with the consideration of additional DG types such as wind power and hydropower and other optimization strategies.

\section{ACKNOWLEDGEMENTS}

The authors would like to thank Universiti Teknologi Brunei for the financial support granted for this research and Berakas Power Management Company Sdn Bhd for providing the necessary data.

\section{REFERENCES}

[1] V. V. Tyagi, N. A. A. Rahim, N. A. Rahim, and J. A. L. Selvaraj, "Progress in solar PV technology: Research and achievement," Renewable and Sustainable Energy Reviews, vol. 20, pp. 443-461, 2013.

[2] N. Barsoum and C. Z. Lee, "Simulation of power flow and protection of a limited bus grid system with injected solar power," Energy and Power Engineering, vol. 5, no. 1, pp. 59-69, 2013.

[3] A. A. C. Priyangika, W. D. A. S. Wijayapala, and H. M. W. Banda, "The Impact of Distributed Generation on Transmission and Distribution Losses in Sri Lankan Power System," in 2016 Electrical Engineering Conference (EECon), 2016, pp. 54-58.

[4] Y. Hou, J. Magnusson, G. Engdahl, and L. Liljestrand, "Impact on Voltage Rise of PV Generation in Future Swedish Urban Areas with High PV Penetration," in 2014 IEEE International Energy Conference (ENERGYCON), 2014, pp. 904-911.

[5] A. P. Kenneth and K. Folly, "Voltage Rise Issue with High Penetration of Grid Connected PV," IFAC Proceedings Volumes, Vol 47, pp. 4959-4966, 2014.

[6] O. Garfi, H. Aloui, and N. Chaker, "Impacts of photovoltaic power source intermittence on a distribution network," International Journal of Electrical and Computer Engineering, vol. 9, no. 6, pp. 5134-5142, 2019.

[7] N. Ghosh, S. Sharma, and S. Bhattacharjee, "A load flow-based approach for optimum allocation of distributed generation units in the distribution network for voltage improvement and loss minimization," International Journal of Computer Applications, vol. 50, pp. 15-22, 2012.

[8] M. Jamil and A. S. Anees, "Optimal sizing and location of SPV (solar photovoltaic) based MLDG (multiple location distributed generator) in distribution system for loss reduction, voltage profile improvement with economical benefits," Energy, vol. 103, pp. 231-239, 2016.

[9] R. Prenc, D. Škrlec, and V. Komen, "Distributed generation allocation based on average daily load and power production curves," International Journal of Electrical Power \& Energy Systems, vol. 53, pp. 612-622, 2013.

[10] K.-y. Liu, W. Sheng, Y. Liu, X. Meng, and Y. Liu, "Optimal sitting and sizing of DGs in distribution system considering time sequence characteristics of loads and DGs," International Journal of Electrical Power \& Energy Systems, vol. 69, pp. 430-440, 2015.

[11] D. Q. Hung, N. Mithulananthan, and R. C. Bansal, "Analytical strategies for renewable distributed generation integration considering energy loss minimization," Applied Energy, vol. 105, pp. 75-85, 2013.

[12] I. Abdelmotteleb, T. Gómez, and J. P. Chaves-Avila, "Benefits of PV Inverter Volt-var Control on Distribution Network Operation," in 2017 IEEE Manchester PowerTech, 2017, pp. 1-6.

[13] A. S. Bouhouras, T. A. Papadopoulos, G. C. Christoforidis, G. K. Papagiannis, and D. P. Labridis, "Loss Reduction via Network Reconfigurations in Distribution Networks with Photovoltaic Units Installed," in 2013 10th International Conference on the European Energy Market (EEM), 2013, pp. 1-8.

[14] G. V. K. Murthy, Sivanagaraju, S. Satyanarayana, and B. H. Rao, "Artificial bee colony algorithm for distribution feeder reconfiguration with distributed generation," International Journal of Engineering Sciences \& Emerging Technologies, vol. 3, no. 2, pp. 50-59, 2012.

[15] S. Ghosh, S. P. Ghoshal, and S. Ghosh, "Optimal sizing and placement of distributed generation in a network system," International Journal of Electrical Power \& Energy Systems, vol. 32, pp. 849-856, 2010.

[16] A. Marneni, A. Kulkarni, and T. Ananthapadmanabha, "Loss Reduction and Voltage Profile Improvement in a Rural Distribution Feeder Using Solar Photovoltaic Generation and Rural Distribution Feeder Optimization Using HOMER,” Procedia Technology, Vol 21, pp. 507-513, 2015.

[17] S. Y. Reddy, D. S. Reddy, and G. K. Rao, "Optimal siting and sizing of solar power sources in interconnection grid system," Indian Journal of Science and Technology, vol. 9, no. 12, pp. 1-9, 2016. 
[18] L. F. Ochoa, A. Padilha-Feltrin, and G. P. Harrison, "Evaluating distributed time-varying generation through a multiobjective index," IEEE Transactions on Power Delivery, vol. 23, no. 2, pp. 1132-1138, 2008.

[19] O. A. Afolabi, W. H. Ali, P. Cofie, J. Fuller, P. Obiomon, and E. S. Kolawole, "Analysis of the load flow problem in power system planning studies," Energy and Power Engineering, vol. 7, no. 10, pp. 509-523, 2015. 